\title{
Efficacy Study of Metronomic Chemotherapy in Metastatic Triple Negative Breast Cancer and Correlation with VEGF, TSP Levels
}

\author{
B. J. Srinivasa ${ }^{1}$, Lalkota Prakash Bhanu${ }^{1}$, Vidhya H. Veldore ${ }^{2}$, \\ R. Raghavendra ${ }^{3}$, Radheshyam Naik ${ }^{1}$ \\ ${ }^{1}$ Department of Medical Oncology, HCG Cancer Specialty Center, Bangalore, India \\ ${ }^{2}$ Department of Histopathology, Molecular Pathology \& Cytogenetics, Triesta Sciences R\&D (A Unit of HCG Cancer Specialty \\ Center), Bangalore, India \\ ${ }^{3}$ Center for Academics and Research, HCG Foundation, Bangalore, India \\ Email: *sripav77@yahoo.co.in
}

How to cite this paper: Srinivasa, B.J., Bhanu, L.P., Veldore, V.H., Raghavendra, R. and Naik, R. (2018) Efficacy Study of Metronomic Chemotherapy in Metastatic Triple Negative Breast Cancer and Correlation with VEGF, TSP Levels. Advances in Breast Cancer Research, 7, 107-119.

https://doi.org/10.4236/abcr.2018.72007

Received: February 6, 2018

Accepted: April 10, 2018

Published: April 13, 2018

Copyright $(9) 2018$ by authors and Scientific Research Publishing Inc. This work is licensed under the Creative Commons Attribution International License (CC BY 4.0).

http://creativecommons.org/licenses/by/4.0/

\begin{abstract}
Background: Treatment refractory metastatic breast cancer patients are at best treated palliatively. We evaluated the effects of metronomic chemotherapy on survival outcomes in this population. Methods: Twenty eight subjects with treatment refractory $(\mathrm{n}=21)$ and treatment naive $(\mathrm{n}=7)$ MBC were included in an open label single arm efficacy study of metronomic chemotherapy. Patients were given a chemotherapy regimen of Tab. Cyclophosphamide $50 \mathrm{mg}$ once daily and Tab. Methotrexate $2.5 \mathrm{mg}$ twice in a week over a minimum period of 3 months or until the progression of their disease whichever was earlier. Monitoring of serum VEGF and Thrombospondin levels were done to correlate the response rates. Data were analysed using chi square test for proportions and Kaplan Meir Survival analysis. Results: The mean age of the study population was $51.5 \pm 14.2$ years. The mean duration of metronomic chemotherapy was $123.89 \pm 97.6$ days. Overall $71.4 \%$ had progressive disease and $28.6 \%$ had stable disease. $55.6 \%$ with treatment naive metastatic breast cancer had stable disease compared to $15.8 \%$ of treatment refractory metastatic breast cancer. There was also a significant improvement in progression free survival in those with tumor load less than $5 \mathrm{cms}$ compared to $>5 \mathrm{cms}$ and in grade 2 compared to grade 3 disease. There was no correlation of serum VEGF levels before and after chemotherapy. There is no significant decrease in TSP levels. Conclusion: The results suggest stable response in one third of study patients. Performance status and tumor load are important predictors in this category of population. There is no significant correlation of serum VEGF and TSP levels before and after chemotherapy. Also, there was
\end{abstract}


no significant correlation of biomarker levels in responding and non-responding patients.

\section{Keywords}

Metronomic Chemotherapy, MBC, Cyclophosphamide, Methotrexate

\section{Introduction}

Metronomic chemotherapy is a continuous or frequent administration of cytotoxic agents at low doses, without extended breaks in between [1]. Conventional chemotherapy is given once in every three weeks or two weeks at maximum tolerated dose with drug free interval to allow normal proliferating cells to recover. Hence, metronomic chemotherapy is said to have low or no toxicity to normal proliferating cells with selective activity against tumor vasculature.

It works by inhibiting the tumor angiogenesis, mainly by selective inhibition of tumor endothelial proliferation, inducing endogenous angiogenesis inhibitor-thrombospondin levels and preventing mobilization of circulating endothelial progenitor cells from bone marrow. It also acts by immunomodulation by selective depletion of TREG cells. Tumor dormancy or direct cytotoxicity is other postulated mechanism of action of metronomic chemotherapy. Many cytotoxic drugs have this property of anti-angiogenesis including drugs used in this study [2].

Preclinical [3] [4] [5] and clinical studies [6] [7] [8] have established the benefits of metronomic chemotherapy in metastatic breast cancer. Oral chemotherapy drugs, used in our study-cyclophosphamide [5] and methotrexate [9] in breast cancer cause selective inhibition of endothelial proliferation and induce apoptosis. Toxicity of this therapy is mild and will not give treatment interruptions as in conventional dosing. Various biomarkers of angiogenesis for response, predicting or to prognosticate the disease have been described but no validated surrogate markers exist.

Triple negative breast cancer (TNBC) constitutes around $15 \%$ of all breast cancer patients [10]. This sub type of breast cancer has no expression of oestrogen (ER), progesterone (PR) and Her2 neu receptors on immunohistochemical analysis. It is more commonly diagnosed in women with less than 40 years of age compared to other subtypes. Pathologically, they are high grade and infiltrating duct carcinoma, and have geographic necrosis and pushing border of invasion [11]. Basal-like type is more common molecular phenotype in TNBC, caludin-low [10], interferon-rich, and luminal-androgen are other subtypes being observed by gene expression analysis [12].

It is biologically aggressive and though it responds well to chemotherapy initially, ultimate prognosis is dismal. Clinical course is also aggressive in both early and metastatic settings [13]. These patients have higher relapse rate with increased risk of loco regional recurrence including lung and brain metastasis. It 
has worst breast cancer specific survival, worse overall survival and increase in death within two years of diagnosis, among all breast cancer [14].

Patients of metastatic triple negative breast cancer visiting/referred to our centre, were offered metronomic chemotherapy.

\section{Patients and Methods}

\subsection{Study Design}

It is a prospective, single arm, efficacy study of metronomic chemotherapy drugs in metastatic triple negative breast cancer patients. After ethical committee clearance, 28 Patients in Metastatic Breast Cancer were enrolled in the study from May 2011 to December 2012.

\subsection{Study Population}

Inclusion criteria:

1) Patients $>18$ years of age.

2) Histologically confirmed invasive breast cancer.

3) Metastatic disease.

4) ER negative, PR negative, HER2 neu negative (Triple negative).

5) ER negative, PR negative but HER2 positive and not on any anti HER2 agents in breast cancer patient.

6) Measurable disease, as defined by RECIST [15] criteria.

7) ECOG performance status (PS) 0 - 2 (Karnofsky PS 60\% - 100\%)

8) Life expectancy $>6$ months.

9) Leukocytes $\geq 3000 / \mu \mathrm{L}$, Absolute neutrophil count $\geq 1500 / \mu \mathrm{L}$, Platelet count $\geq 100,000 / \mu \mathrm{L}$, hemoglobin $>9.0 \mathrm{~g} / \mathrm{dL}$.

10) Adequate hepatic function, defined as follows: total bilirubin $<1.5 \times$ ULN; aspartate aminotransferase (AST) $<3 \times$ ULN (or $<5 \times$ ULN if liver metastases); alanine aminotransferase (ALT) $<3 \times$ ULN (or $\leq 5 \times$ ULN if liver metastases).

11) Creatinine normal OR creatinine clearance $\geq 60 \mathrm{~mL} / \mathrm{min}$.

Women of childbearing potential must agree to use adequate contraception (as per institutional standard of care) during treatment and until 6 months after the last administration of investigational drugs.

\subsection{Exclusion Criteria}

1) Women who are pregnant or breastfeeding or patient of childbearing potential without being tested for pregnancy at baseline or with a positive test.

2) Those are on hormonal or other antiangiogenic therapies.

3) Presence of exclusively non-measurable disease (I/E: exclusive bone disease with non-representative tumor markers).

4) Unwillingness or inability to comply with study requirements.

5) Other concurrent investigational agents within 3 months of starting the therapy.

6) Concurrent combination anti-retroviral therapy for HIV-positive patients. 
Medicinal product (dose/route/regimen):

Tablet cyclophosphamide $50 \mathrm{mg}$ per day daily.

Tablet methotrexate $2.5 \mathrm{mg}$ for 2 days in a week (Day 1 and Day 4).

Duration of Study: Each patient was given treatment for 3 months or till progression/occurrence of Grade 3 or more of haematological toxicity or Grade 2 or more of non-haematological toxicity. Follow up of all the patients will be done every month for 6 months or till any adverse event, whichever is earlier. Responding patients were continued on therapy for another 3 months or till progression of the disease.

Tumour assessments: was performed at baseline, 3rd month, and at 6th month (if response is documented at 3rd month) based on RECIST criteria (version 1.1). Assessments were done using CT chest, abdomen and MRI brain (if required or documented CNS metastasis previously). Complete remission (CR) was defined as disappearance of all target lesions. Partial Response (PR) was defined as at least a $30 \%$ decrease in the sum of diameters of target lesions. Progressive Disease (PD) was defined as at least a $20 \%$ increase in the sum of diameters of target lesions. Stable Disease (SD) was defined as neither sufficient shrinkage to qualify for PR nor sufficient increase to qualify for PD, taking as reference the smallest sum diameters while on study. Clinical and laboratory AEs will be reported and graded according to NCI-CTCAE version 4.0 [16].

\subsection{Biomarker Assessment}

ELISA based analysis was carried out in sera for VEGF and thrombospondin (TSP-1) at baseline and 3rd month. Test was carried out in pre and post chemotherapy samples of 16 ( 8 of them were in doublets) breast cancer patients. $10 \mathrm{ml}$ of peripheral blood was collected in plain vacationer and centrifuged for $10 \mathrm{~min}$. Aliquot samples were then stored at $-800 \mathrm{C}$ until analysis was performed.

Serum VEGF and serum thrombospondin (TSP-1) levels were assessed using QuantikineR ELISA kit, R\&D systems, Minneapolis, USA. Assay is based on the principle of quantitative sandwich enzyme immunoassay technique. A monoclonal antibody specific for VEGF or thrombospondin has been pre-coated onto a micro plate. Standards and samples are pipetted into the wells and any VEGF or thrombospondin is bound by the immobilized antibody. After washing away any unbound substances, an enzyme-linked polyclonal antibody specific for VEGF or thrombospondin is added to the wells. Following a wash to remove any unbound antibody-enzyme reagent, a substrate solution is added to the wells and colour develops in proportion to the amount of VEGF or thrombospondin bound in the initial step. The colour development is stopped and the intensity of the colour is measured.

Each marker assessment was done separately and methods were followed strictly as per protocol mentioned in the booklet. Optical density of each well determined at 5, 15 and 30 minute intervals using micro plate reader set at 492 $\mathrm{nm}$ and $620 \mathrm{~nm}$. A standard curve is plotted using the mean absorbance for each standard on the $\mathrm{y}$-axis against the concentration on the $\mathrm{x}$-axis and by drawing a 
best fit curve through the points on the graph.

\subsection{Statistical Analysis}

Data were analyzed using SPSS version 16 for windows. Descriptive statistics using proportions were analyzed by frequencies and percentage. Pearson Chi-square analysis was used to analyze the effect of predictor variables such as T size, stage, Her 2 neu status on response rates. Kaplan Meier survival analysis was carried out with predictor variables such as stage, Her 2 neu status, tumor load, performance status, pathologic grade for disease free and overall survival. Paired $t$ test was used to analyze VEGF and thrombospondin levels and independent samples test is used to analyze changes in VEGF and thrombospondin levels following metronomic chemotherapy between responders and non-responders following metronomic chemotherapy.

\section{Results}

Twenty eight patients were enrolled for the study from May 2011 to December 2012. The mean age of the study population was $51.5 \pm 14.2$ years. Seven patients were more than 65 years old (Table 1 ).

Response: The mean duration of metronomic chemotherapy was $123.89 \pm$ 97.6 days. Overall 8 patients $(28.6 \%)$ had stable disease and 20 patients $(71.4 \%)$ had progressive disease. There was no complete or partial response. Response rates in different subpopulation of study patients is summarised in Table 2.

\subsection{Response Rates in Metastatic Triple Negative Breast Cancer (Figure 1, Figure 2)}

Survival: Mean duration of survival was 142 days. Thirteen patients (50\%) were alive and 13 patients (50\%) were dead with 2 patients lost to follow up at the end of 20 months.

Mean progression free survival in study population is 115 days. Mean overall survival in patients with ECOG performance status of 1 is 228 (95\% CI 163 to 294 days) days and 70 days (95\% CI 38 to 102 days) in patients with ECOG 2 (p $=0.05$ ). Mean overall survival in pathology grade 2 is 321 days (95\% CI 242 to 400 days) and 157 days (95\% CI 92 to 223 days) in grade 3 histology ( $\mathrm{p}=0.03$ ). Median overall survival in patients with tumor load less than $5 \mathrm{cms}$ is 245 days (95\% CI of 171 to 319 days) and 86 days (95\% CI 54 to 118 days) in patients with tumor load $>5 \mathrm{~cm}(\mathrm{p}=0.03)$.

Median Progression free survival in those with tumor load less than $5 \mathrm{~cm}$ is 143 day (95\% CI 126 to 288 days) and 58 days (95\% CI 42 to 108 days) in patients with tumor load more than $>5 \mathrm{~cm}(\mathrm{p}=0.05)$. Mean progression free survival in grade 2 is 287 days (95\% CI 195 to 379 days) and 122 days (95\% CI 60 to 184 days) in grade 3 patients $(p=0.02)$. Survival in those who went on to receive further treatment after progression is 100 days and 95 days in those who did not receive any treatment $(\mathrm{p}=0.935)$ (Figure 3 , Figure 4$)$. 
Table 1. Baseline characteristics.

\begin{tabular}{|c|c|c|}
\hline Strata & No of patients (n) & Percentage \\
\hline Eligible patients & 28 & \\
\hline Median age, years (range) & $51.57(32-80)$ & \\
\hline \multicolumn{3}{|l|}{ Baseline ECOG performance } \\
\hline \multicolumn{3}{|l|}{ Status } \\
\hline 1 & 23 & 82 \\
\hline 2 & 5 & 17 \\
\hline \multicolumn{3}{|l|}{ Post-menopausal } \\
\hline Yes & 21 & 75 \\
\hline No & 7 & 25 \\
\hline \multicolumn{3}{|l|}{ Co morbidities } \\
\hline Yes & 13 & 46.4 \\
\hline No & 15 & 53.57 \\
\hline \multicolumn{3}{|l|}{ Pre treatment } \\
\hline Yes & 21 & 75 \\
\hline No & 7 & 25 \\
\hline \multicolumn{3}{|l|}{ Previous chemotherapy } \\
\hline None & 7 & 25 \\
\hline $1^{\text {st }}$ line & 12 & 42.85 \\
\hline $2^{\text {nd }}$ line or more & 9 & 32.14 \\
\hline
\end{tabular}

Table 2. Responses in metastatic triple negative breast cancer.

\begin{tabular}{|c|c|c|c|c|c|}
\hline Category & $\mathrm{N}$ & $\begin{array}{l}\text { Stable disease } \\
\text { (SD) } \mathrm{n}(\%)\end{array}$ & $\begin{array}{c}\text { Progressive } \\
\text { disease (PD) n (\%) }\end{array}$ & $\begin{array}{l}\text { Chi-squa } \\
\text { re value }\end{array}$ & $\mathrm{p}$ value \\
\hline Overall response & 28 & $8(28.6)$ & $20(71.4)$ & & \\
\hline Chemo naïve & 7 & $4(55.6)$ & $3(44.4)$ & 4.7 & 0.03 \\
\hline $\begin{array}{l}\text { Chemo refractory } \\
\text { (received one or two } \\
\text { lines of chemotherapy) }\end{array}$ & 21 & $4(15.8)$ & $17(84.2)$ & & \\
\hline Triple negative & 20 & $7(35)$ & $13(65)$ & 7.605 & 0.02 \\
\hline $\begin{array}{c}\text { ER neg, PR neg, HER2 } \\
\text { neu positive }\end{array}$ & 7 & $0(0)$ & $7(100)$ & & \\
\hline $\begin{array}{c}\text { Unknown receptor } \\
\text { status }\end{array}$ & 1 & $1(100)$ & $0(0)$ & & \\
\hline Tumor load $<5 \mathrm{~cm}$ & 17 & $7(25)$ & $10(35.7)$ & 3.369 & 0.066 \\
\hline Tumor load $>5 \mathrm{~cm}$ & 10 & $1(12.5)$ & $10(50)$ & & \\
\hline Pathological grade 2 & 9 & $3(10.7)$ & $6(21.4)$ & 0.147 & 0.70 \\
\hline Pathological grade 3 & 19 & $5(62.5)$ & $14(70)$ & & \\
\hline ECOG PS 1 & 23 & $7(25)$ & $16(57.1)$ & 0.219 & 0.64 \\
\hline ECOG PS 2 & 5 & $1(3.6)$ & $4(14.3 \%)$ & & \\
\hline
\end{tabular}

Toxicity: There was no observed haematological toxicity, GI toxicity in study population. There were no treatment delays or interruption because of toxicity. At each follow up, most of the patients had complaint of tiredness. But one 


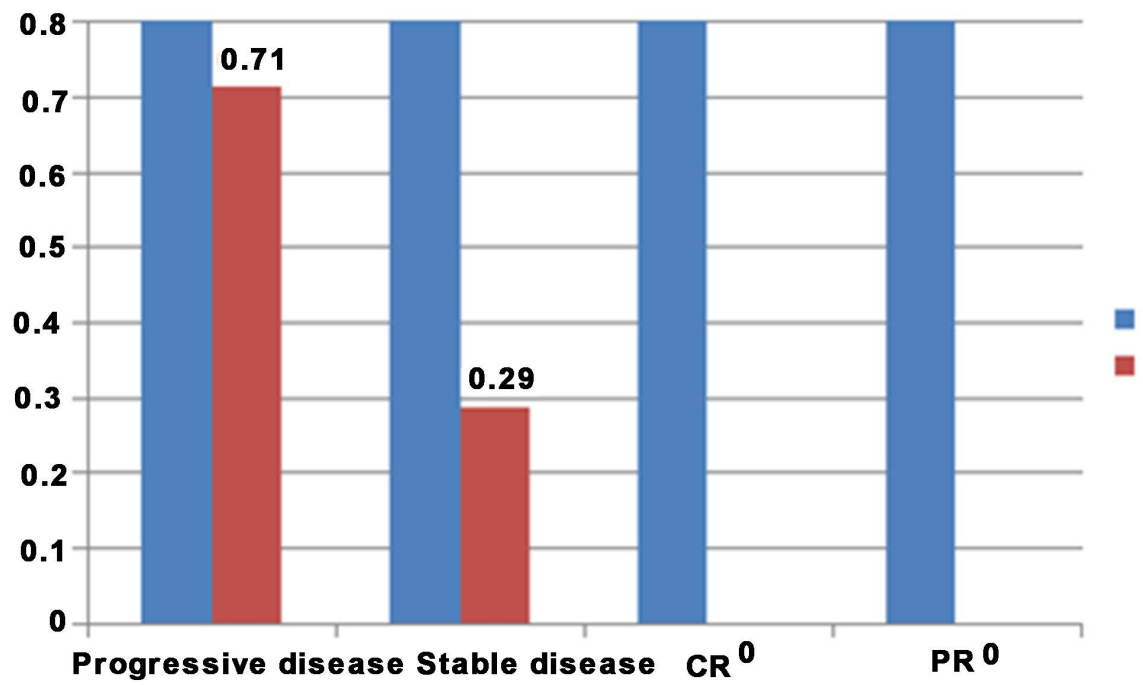

Figure 1. Response as per RECIST criteria.

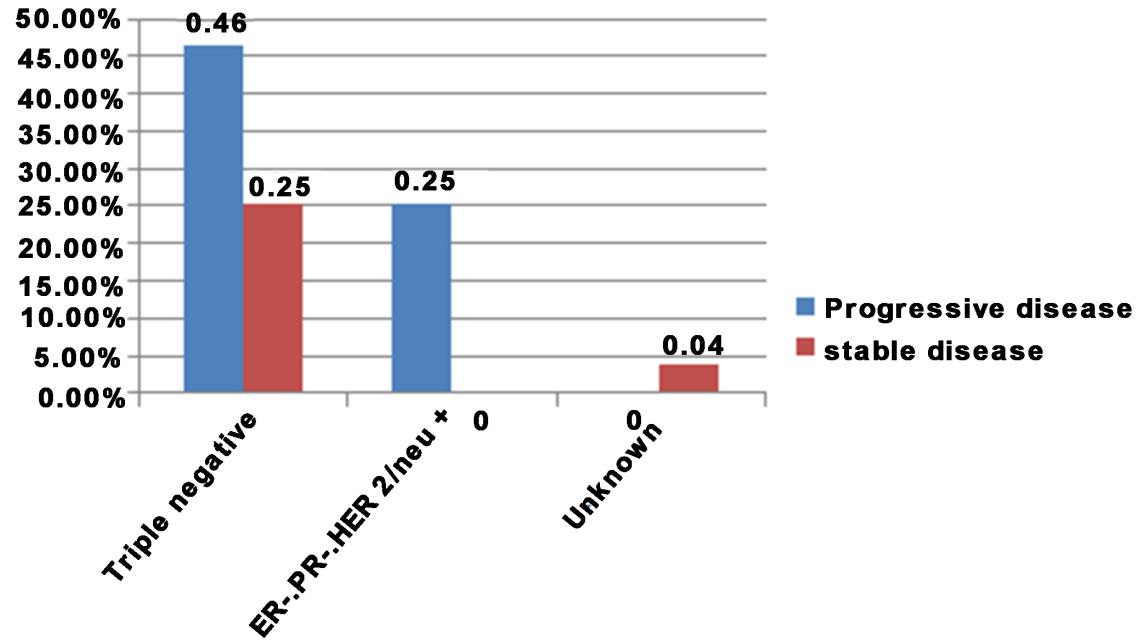

Figure 2. Response rate according to hormonal status.

patient developed MDS transformed AML, after 6 months of completion of metronomic chemotherapy (Figure 5, Figure 6).

Three months after the diagnosis, MDS transformed to AML, with peripheral smear showing $86 \%$ blasts. Patient was managed with supportive treatment and she succumbed to death one month later.

\subsection{Biomarkers of Angiogenesis}

Serum VEGF and Thrombospondin levels in metastatic triple negative breast cancer at baseline and 3 months after treatment are summarised in the following tables (Tables 3-5).

\section{Discussion}

Treatment of metastatic triple negative breast cancer patients with oral cyclophosphamide $50 \mathrm{mg}$ daily and methotrexate $2.5 \mathrm{mg}$ twice in a week given for 


\section{Survival Functions}

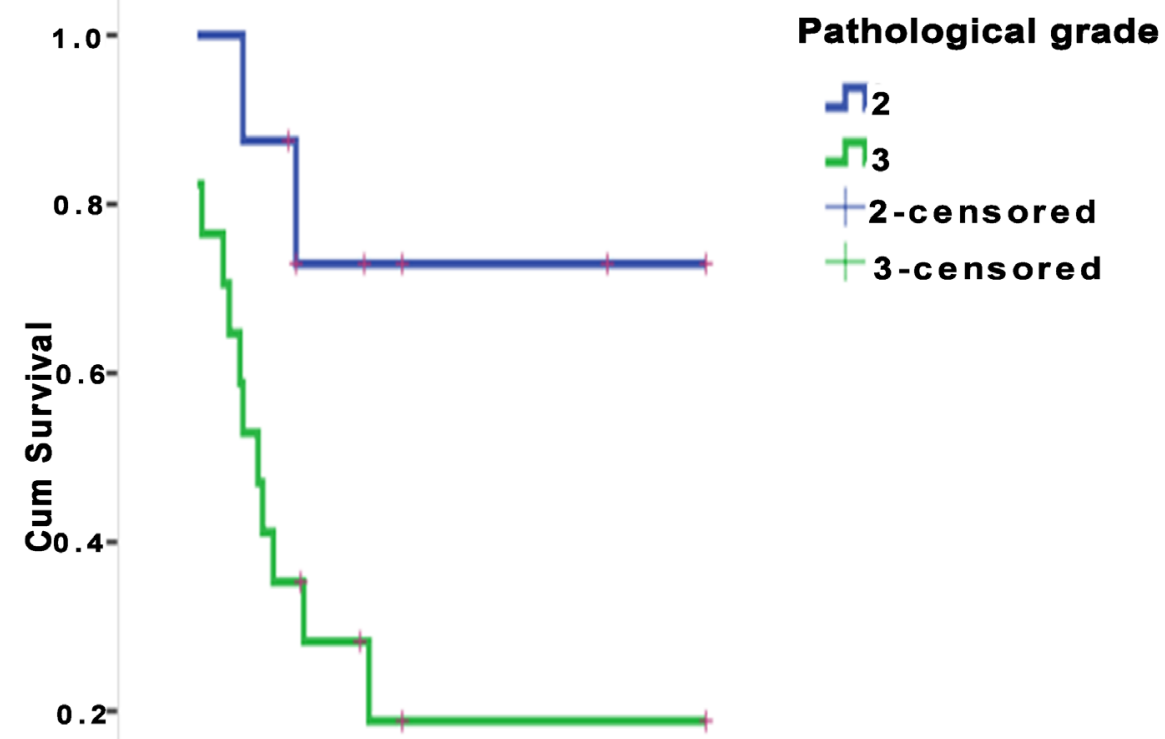

0.0-

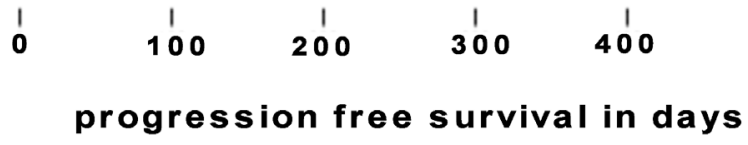

Figure 3. PFS and pathological grade. Pathological grade 2 (Median 95 vs 70 days) Path grade 3 Log rank 5.184, $\mathrm{p}=0.02$.

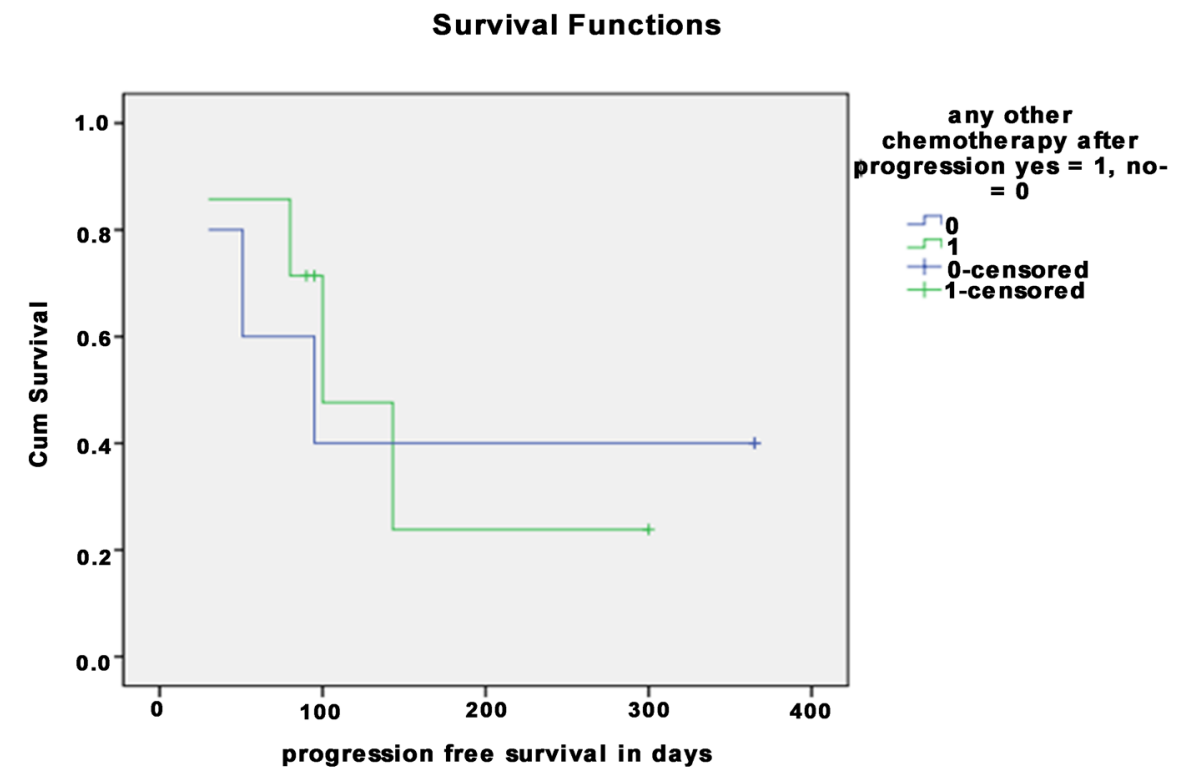

Figure 4. PFS of patients receiving second line chemotherapy. Second line chemotherapy after progression (median 95 day vs 100 days) No other chemotherapy. Log rank 0.007 (p $=0.935)$. 


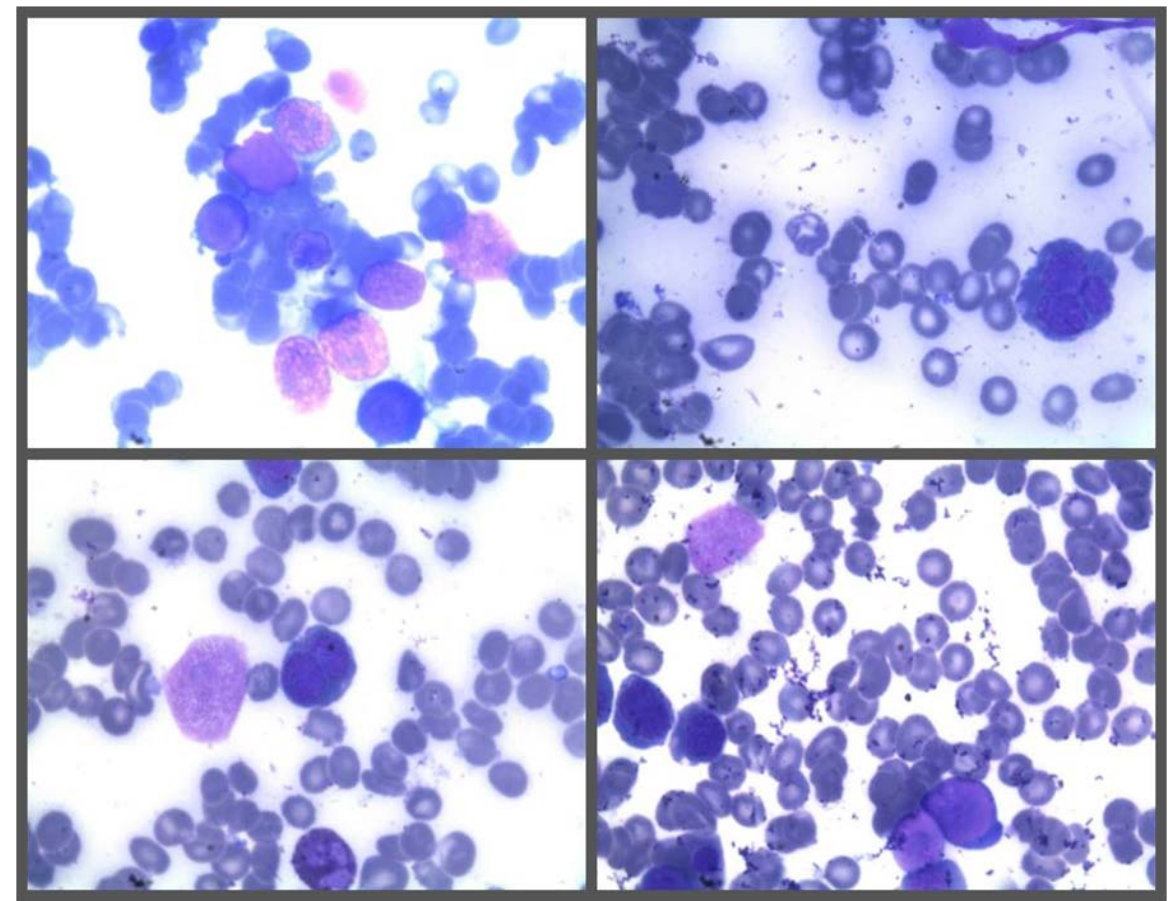

Figure 5. Bone marrow aspiration smear study of a patient aged 55 years, who developed MDS-RAEB II, 3 months after the completion of metronomic chemotherapy for breast cancer. This patient has received CMF regimen 10 year back. Study showing dyserythropoietic cells (red arrowheads) with bizzare, multinucleated cells, internuclear bridging, and about $15 \%$ blasts (black arrows) with high N/C ratio.

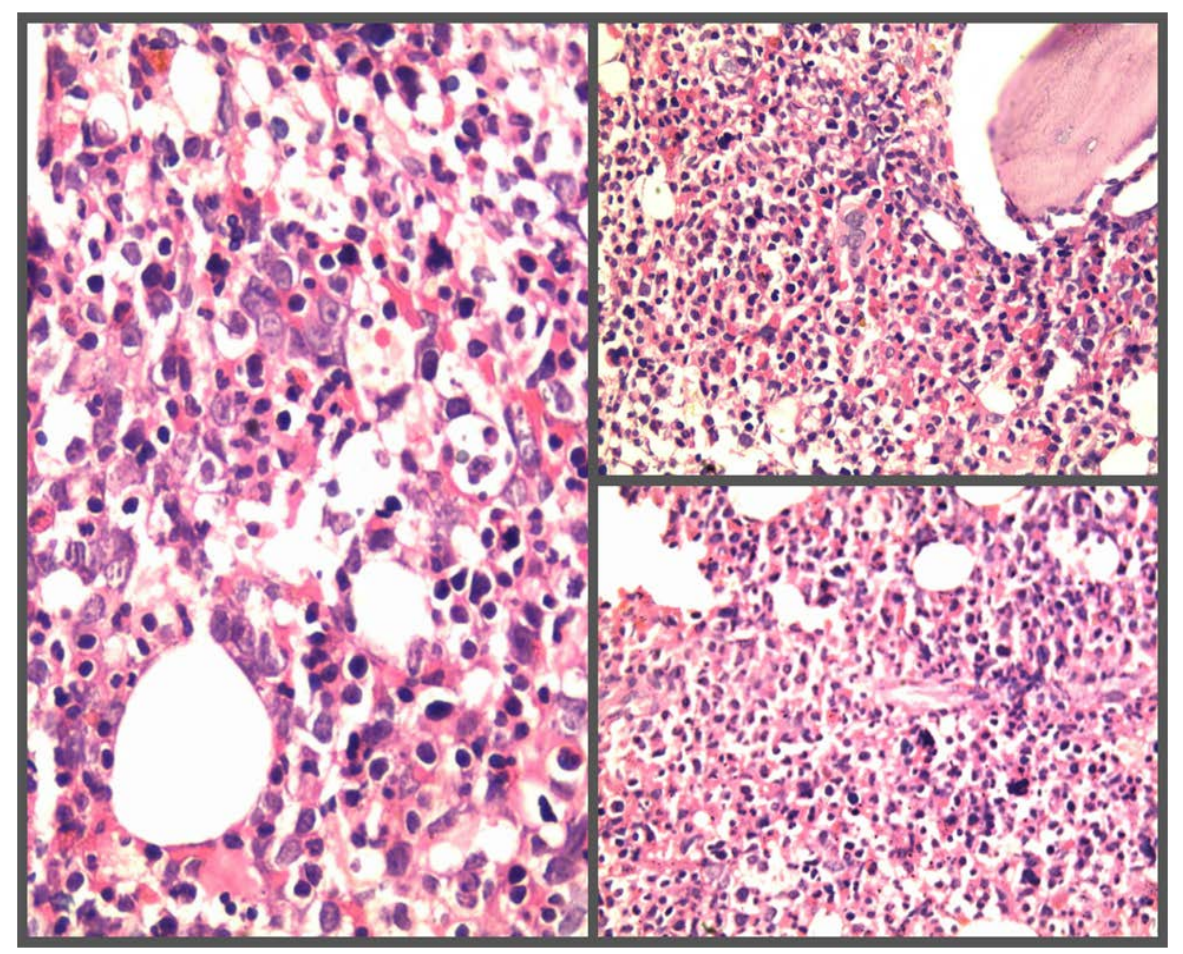

Figure 6. Bone marrow biopsy of same patient showing hyper cellular marrow, dysplastic megakaryocytes (red arrow head), myeloblasts (blue arrow), and erythroid hyperplasia exhibiting dysplasia (black arrow). 
Table 3. Results of VEGF and thrombospondin (TSP) levels in breast cancer.

\begin{tabular}{ccccccc}
\hline $\begin{array}{c}\text { Serum } \\
\text { biomarker }\end{array}$ & $\mathrm{N}$ & $\begin{array}{c}\text { Pre chemo in } \\
\mathrm{pg} / \mathrm{ml} \\
(\text { Mean } \pm \mathrm{SD})\end{array}$ & $\begin{array}{c}\text { Post chemo } \\
\text { in } \mathrm{pg} / \mathrm{ml} \\
(\mathrm{Mean} \pm \mathrm{SD})\end{array}$ & $\begin{array}{c}\text { Difference } \\
(\text { Mean } \pm \mathrm{SD})\end{array}$ & $\mathrm{t}$ Test & $\mathrm{p}$ value \\
\hline VEGF & 16 & $133.3 \pm 70.6$ & $145 \pm 87$ & $12.5 \pm 93.4$ & -0.537 & 0.59 \\
TSP & 16 & $86 \pm 34$ & $67 \pm 42$ & $18.7 \pm 62.5$ & 1.20 & 0.24 \\
\hline
\end{tabular}

Table 4. Serum VEGF levels according to response.

\begin{tabular}{ccccccc}
\hline $\begin{array}{c}\text { Response } \\
\text { category }\end{array}$ & $\mathrm{N}$ & $\begin{array}{c}\text { Pre chemo } \\
\text { VEG levels in } \\
\mathrm{pg} / \mathrm{ml} \\
(\mathrm{Mean} \pm \mathrm{SD})\end{array}$ & $\begin{array}{c}\text { Post chemo } \\
\text { VEGF levels } \\
\text { in pg/ml } \\
(\text { Mean } \pm \mathrm{SD})\end{array}$ & $\begin{array}{c}\text { Percentage of } \\
\text { change }\end{array}$ & t Test & $\mathrm{p}$ value \\
\hline $\begin{array}{c}\text { Progressive } \\
\text { disease }\end{array}$ & 9 & $114 \pm 63$ & $123 \pm 82$ & -8.9378 & -0.170 & 0.86 \\
Stable disease & 7 & $157 \pm 76$ & $175 \pm 91$ & -17.2100 & & \\
\hline
\end{tabular}

Table 5. Serum thrombospondin (TSP) levels according to response.

\begin{tabular}{ccccccc}
\hline $\begin{array}{c}\text { Response } \\
\text { category }\end{array}$ & $\mathrm{N}$ & $\begin{array}{c}\text { Pre chemo TSP } \\
\text { levels in pg/ml } \\
(\text { Mean } \pm \text { SD })\end{array}$ & $\begin{array}{c}\text { Post chemo TSP } \\
\text { levels in pg/ml } \\
(\text { Mean } \pm \text { SD })\end{array}$ & $\begin{array}{c}\text { Percentage of } \\
\text { change }\end{array}$ & t Test & p value \\
\hline $\begin{array}{c}\text { Progressive } \\
\text { disease }\end{array}$ & 9 & $98 \pm 25$ & $68 \pm 38$ & 30.2656 & -0.824 & 0.42 \\
Stable disease & 7 & $70 \pm 39$ & $66 \pm 50$ & 3.9971 & \\
\hline
\end{tabular}

three months to 28 patients. Results suggest stable disease as the main response in $28.6 \%$ of study population without any complete or partial remission. Response is more in chemo naïve patients than in chemo refractory patients. There is no improved response in other sub groups of patients with ECOG 1 performance status (PS), with lesser tumor burden patients and pathological grade 2 patients.

Median progression free survival of study population is around 4 months $\mathrm{Pa}$ tients with ECOG 1 PS had significant improvement in overall survival compared to patients with ECOG 2 PS. Overall survival and progression free survival was improved significantly in patients with pathological grade 2 compared to grade 3. Higher tumor burden patients (sum of all target lesion $>5 \mathrm{~cm}$ ) had inferior overall survival and progression free survival compared to patients with lower tumor burden (sum of all target lesion $<5 \mathrm{~cm}$ ). There were no acute toxicity and no interruption of treatment.

There is no significant correlation of serum VEGF levels before and after chemotherapy. Levels increased after therapy rather than expected decline. Raised levels were also not significant. There was no significant decrease in TSP levels after therapy. Also, there was no significant correlation of biomarker levels in responding and non-responding patients.

Colleoni et al. (2002) [6] reported overall response rate (CR + PR) 19\% and an overall clinical benefit (CR + PR + SD > 24 weeks) 31.7\%, in 63 patients treated 
with above combination. Colleoni et al. (2006) [7] reported overall response rate of $20.9 \%$ and an overall clinical benefit of $41.5 \%$ in 171 patients treated with same combination but with or without addition of thalidomide, though addition of thalidomide did not influence any results.

Objective response in this study did not correlate with above studies but disease stabilization response is comparable. Both the above studies comprised of hormone positive and hormone negative patients and were not selective for triple negative subtype as in this study. Analysis of above two studies by Orland et al. (2006) [8] noted more benefit in hormone receptor positive patients. Hormone responsive tumors are slow growing and this might have influenced the results. Hence, response rate observed in this poor prognostic group in our study is encouraging. Improved response in chemo naïve patients in this study as also observed in other studies indicate that initially tumors are dependent on angiogenesis for their growth and hence inhibited by metronomic chemotherapy. Repeated administration of cytotoxic drugs at conventional doses impairs tumor vasculature and growth may not be entirely dependent on angiogenesis.

Cumulative dose of cyclophosphamide achieved in this study is $150 \%$ more compared to the same drug given in AC regimen for 4 cycles in 3 months. This indicates that though low dose used is not actually very low but low dose given in dose dense form over 3 months. This is achieved with minimal toxicity even in previously heavily pretreated patients.

Tumor burden parameter is taken for assessment to see whether the patient with small amount of disease responds better or not. And this is shown by improved survival in low burden patients indicating that smaller the tumor, lesser the heterogeneity and resistance (Figure 7).

Though acute toxicity observed is nil, one patient developing MDS transformed AML is a cause for concern. This patient was treated with CMF regimen 10 year back as an adjuvant regimen. Possibility of her current metronomic regimen containing alkylating agent, enhancing the already existing risk of leukemia by previous exposure cannot be excluded.

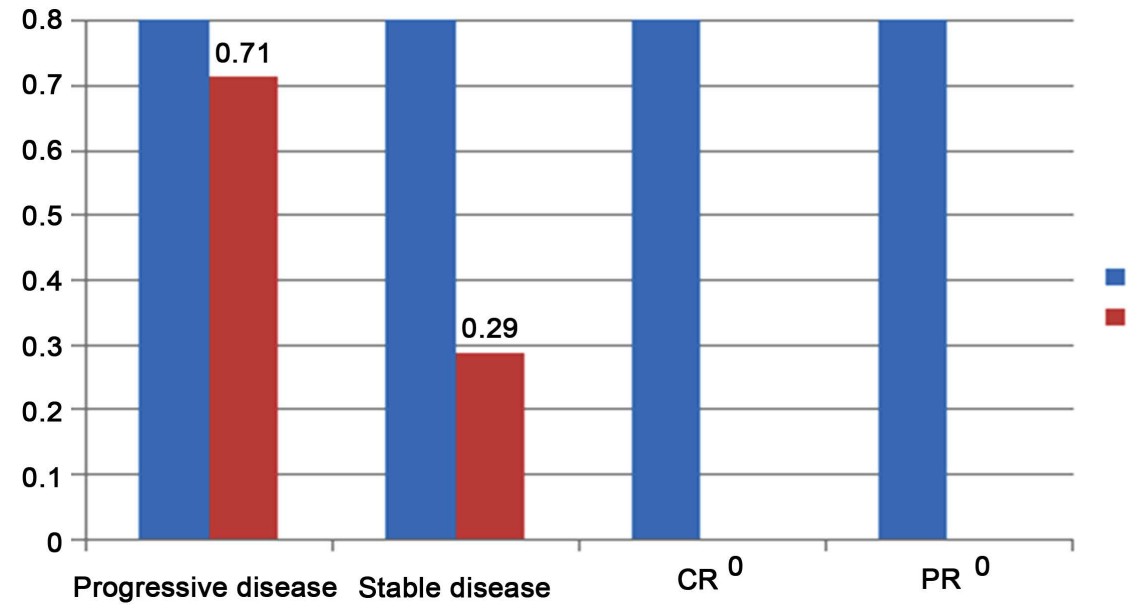

Figure 7. Comparison with others studies. 
Limitation of the study is smaller sample size, no control arm and failure to assess quality of life which is more useful in terms of assessment of effectiveness of therapy in palliative setting. And one fourth of the study population also had one more poor prognostic subtype of ER negative, PR negative and HER 2/neu positive breast cancer. Two poor prognostic groups might have influenced the response rates.

\section{Conclusion}

Metronomic chemotherapy in metastatic triple negative breast cancer patients can be considered as an effective option. It is to be considered early in the disease, when the tumor burden is low. Performance status, pathological grade and tumor load are important predictors in this category of population with improved progression free and overall survival. Serum VEGF levels and Thrombospondin levels did not correlate with response and anti-angiogenic effect of metronomic chemotherapy in both study population. Metronomic chemotherapy approach is safe with no or minimal toxicity in study population. As the sample size is small, above results require validation in randomized control clinical studies.

\section{References}

[1] André, N., Banavali, S., Snihur, Y. and Pasquier, E. (2013) Has the Time Come for Metronomics in Low-Income and Middle-Income Countries? The Lancet Oncology, 14, e239. https://doi.org/10.1016/S1470-2045(13)70056-1

[2] Pasquier, E., Kavallaris, M. and André, N. (2010) Metronomic Chemotherapy: New Rationale for New Directions. Nature Reviews Clinical Oncology, 7, 455-465. https://doi.org/10.1038/nrclinonc.2010.82

[3] Browder, T., Butterfield, C.E., Kräling, B.M., Shi, B., Marshall, B., O’Reilly, M.S., et al. (2000) Antiangiogenic Scheduling of Chemotherapy Improves Efficacy against Experimental Drug-Resistant Cancer. Cancer Research, 60, 1878-1786.

[4] Klement, G., Huang, P., Mayer, B., Green, S.K., Man, S., Bohlen, P., Hicklin, D. and Kerbel, R.S. (2002) Differences in Therapeutic Indexes of Combination Metronomic Chemotherapy and Anti-VEGFR-2 Antibody in Multidrug-Resistant Human Breast Cancer Xenografts. Clinical Cancer Research, 8, 221-232.

[5] Man, S., Bocci, G., Francia, G., Green, S.K., Jothy, S., Hanahan, D., et al. (2002) Antitumor Effects in Mice of Low-Dose (Metronomic) Cyclophosphamide Administered Continuously through the Drinking Water. Cancer Research, 62, 2731-2735.

[6] Colleoni, M., Rocca, A., Sandri, M.T., Zorzino, L., Masci, G., Nolè, F., et al. (2002) Low-Dose Oral Methotrexate and Cyclophosphamide in Metastatic Breast Cancer: Antitumor Activity and Correlation with Vascular Endothelial Growth Factor Levels. Annals of Oncology, 13, 73-80. https://doi.org/10.1093/annonc/mdf013

[7] Colleoni, M., Orlando, L., Sanna, G., Rocca, A., Maisonneuve, P., Peruzzotti, G., et al. (2006) Metronomic Low-Dose Oral Cyclophosphamide and Methotrexate Plus or Minus Thalidomide in Metastatic Breast Cancer: Antitumor Activity and Biological Effects. Annals of Oncology, 17, 232-238.

https://doi.org/10.1093/annonc/mdj066

[8] Orlando, L., Cardillo, A., Rocca, A., Balduzzi, A., Ghisini, R., Peruzzotti, G., et al. 
(2006) Prolonged Clinical Benefit with Metronomic Chemotherapy in Patients with Metastatic Breast Cancer. Anticancer Drugs, 17, 961-967. https://doi.org/10.1097/01.cad.0000224454.46824.fc

[9] Hirata, S., Matsubara, T., Saura, R., Tateishi, H. and Hirohata, K. (1989) Inhibition of In Vitro Vascular Endothelial Cell Proliferation and In Vivo Neovascularization by Low Dose Methotrexate. Arthritis \& Rheumatology, 32, 1065-1073. https://doi.org/10.1002/anr.1780320903

[10] Kaplan, H.G. and Malmgren, J.A. (2008) Impact of Triple Negative Phenotype on Breast Cancer Prognosis. The Breast Journal, 14, 456-463.

https://doi.org/10.1111/j.1524-4741.2008.00622.x

[11] Livasy, C.A., Karaca, G., Nanda, R., Tretiakova, M.S., Olopade, O.I., Moore, D.T., et al. (2006) Phenotypic Evaluation of the Basal-Like Subtype of Invasive Breast Carcinoma. Modern Pathology, 19, 264-271. https://doi.org/10.1038/modpathol.3800528

[12] Anders, C.K. and Carey, L.A. (2013) Epidemiology, Risk Factors and the Clinical Approach to ER/PR Negative, HER2-Negative (Triple-Negative) Breast Cancer (Online). http://www.uptodate.com/contents/epidemiology

[13] DeVita Jr, V.T., Lawrence, T.S. and Rosenberg, S.A., Eds. (2011) Cancer Principles and Practice of Oncology. 9th Edition, Lippincott Williams \& Wilkins, USA, 799, $814,843$.

[14] Groome, P.A., Bolejack, V., Crowley, J.J., Kennedy, C., Krasnik, M., Sobin, L.H., et al. (2007) The IASLC Lung Cancer Staging Project: Validation of the Proposals for Revision of the T, N, and M Descriptors and Consequent Stage Groupings in the Forthcoming (Seventh) Edition of the TNM Classification of Malignant Tumours. Journal of Thoracic Oncology, 2, 694-705. https://doi.org/10.1097/JTO.0b013e31812d05d5

[15] Eisenhauer, E.A., Therasse, P., Bogaerts, J., Schwartz, L.H., Sargent, D., Ford, R., et al. (2009) New Response Evaluation Criteria in Solid Tumours: Revised RECIST Guideline (Version 1.1). European Journal of Cancer, 45, 228-247. https://doi.org/10.1016/j.ejca.2008.10.026

[16] U.S. Department of Health and Human Services, National Institutes of Health, National Cancer Institute (2009) Common Terminology Criteria for Adverse Events (CTCAE) (v4.02: S). 